\title{
THE EVOLUTION OPERATOR APPROACH TO FUNCTIONAL DIFFERENTIAL EQUATIONS WITH DELAY
}

\author{
WOLFGANG M. RUESS
}

(Communicated by Charles Pugh)

\begin{abstract}
The nonlinear nonautonomous functional differential equation $\dot{x}(t) \in B(t) x(t)+F\left(t, x_{t}\right), \quad t \geq s, x_{s}=\varphi$, is considered. The representation of the solution to this equation via the associated evolution operator is extended from the single-valued case to the general case of multivalued operators $B(t)$.
\end{abstract}

\section{INTRODUCTION}

The object of this paper is the evolution operator approach to nonlinear nonautonomous delay functional differential equations of the form

$$
\text { (FDE) }\left\{\begin{array}{l}
\dot{x}(t) \in B(t) x(t)+F\left(t, x_{t}\right), \quad t \geq s, \\
x_{s}=\varphi \in E
\end{array}\right.
$$

and their autonomous counterparts. Here, $E$ denotes a Banach space of functions ("histories") from an interval $I \subset(-\infty, 0]$ into a Banach space $X$, where either $I=(-\infty, 0]$ (infinite delay), or $I=[-r, 0], r>0$ (finite delay), $(B(t))_{t>0}$ is a family of, generally nonlinear and multivalued, operators $B(t) \subset X \times X$, and $(F(t, \cdot))_{t>0}$ is a family of Lipschitz-continuous functions from $E$ into $X$. Finally, for a function $x: I \cup[0, \infty) \rightarrow X$ and $s \in \mathbb{R}^{+}, x_{s}: I \rightarrow X$ is defined by $x_{s}(\theta):=x(s+\theta), \theta \in I$.

For the finite delay case $I=[-r, 0], r>0$, and initial history spaces $E$ consisting of continuous functions, the evolution operator approach to (FDE) consists of basically three steps:

(1) Associate with (FDE) a family $(A(t))_{t \geq 0}$ of m-accretive operators $A(t)$ in $E$, defined by

$$
\left\{\begin{array}{l}
D(A(t))=\left\{\varphi \in E \mid \varphi^{\prime} \in E, \varphi(0) \in D(B(t)),\right. \\
A(t) \varphi:=-\varphi^{\prime},
\end{array}\right.
$$

generating an evolution system $\{U(t, s) \mid 0 \leq s \leq t\}$ in $E$.

Received by the editors September 22, 1991 and, in revised form, March 3, 1992.

1991 Mathematics Subject Classification. Primary 34K15, 34K20, 34K30; Secondary 47H06, $47 \mathrm{H} 20$.

Key words and phrases. Functional differential equations with delay, stability, nonlinear accretive operators, nonlinear evolution systems.

This work was supported in part by the Deutsche Forschungsgemeinschaft (DFG). 
(2) Show that, for certain $\varphi \in E, \quad\{U(t, s) \mid 0 \leq s \leq t\}$ acts as a translation, i.e., if $x(\varphi ; s):[-r, \infty) \rightarrow X$ is defined by

$$
x(\varphi ; s)(t)= \begin{cases}\varphi(t-s), & s-r \leq t \leq s \\ (U(t, s) \varphi)(0), & s \leq t,\end{cases}
$$

then $U(t, s) \varphi=x_{t}(\varphi ; s)$ for $t \geq s$.

(3) Show that, for certain $\varphi \in E$, the function $x(\varphi ; s)$ defined in (2) is actually a solution to (FDE).

This program has been developed in a series of papers, with the objective to bring into play the well-developed generation theory of nonlinear evolution systems for a study of stability results for solutions to (FDE); cf. [2-5, 12-15, 18, 20-22]. Steps (1) and (2) have been worked out for (or can easily be adapted to) the case of multivalued operators $(B(t))_{t \geq 0}$, whereas step (3) has been restricted to the case of the operators $B(t)$ being single-valued, thus excluding the multivalued case that arises naturally in concrete problems (compare $\S 3$ ).

The object of this paper is to close this gap and to carry out step (3) in the multivalued case.

After a preliminary section, setting up the problem in detail, we prove the main technical result in $\S 2$ and turn to applications to various forms of (FDE) in $\S 3$.

\section{Preliminaries and statement of the Problem}

Throughout the paper, $X$ will denote a (real) Banach space. Given a subset $D$ of a Banach space $Y$, $\operatorname{co} D$ will denote its convex hull, and $\operatorname{cl} D$ its (norm-) closure in $Y$. Recall [8] that a subset $A \subset Y \times Y$ is said to be accretive in $Y$ if for each $\lambda>0$ and each pair $\left[x_{i}, y_{i}\right] \in A, i \in\{1,2\}$, we have

$$
\left\|\left(x_{1}+\lambda y_{1}\right)-\left(x_{2}+\lambda y_{2}\right)\right\| \geq\left\|x_{1}-x_{2}\right\|,
$$

and $m$-accretive in $Y$ if, in addition, $R(I+\lambda A)=Y$ for all $\lambda>0$. Moreover, for $\lambda>0, \quad J_{\lambda}=(I+\lambda A)^{-1}$ and $A_{\lambda}=\lambda^{-1}\left(I-J_{\lambda}\right)$. For all these notions and the general theory of accretive sets and evolution equations, the reader is referred to $[1,8,17]$. if

A continuous function $x: I \cup[0, \infty) \rightarrow X$ is said to be a solution to (FDE),

(a) $x_{s}(\theta)=\varphi(\theta)$ for $\theta \in I$,

(b) $\left.x\right|_{[s, \infty)}$ is locally absolutely continuous and differentiable a.e. on $[s, \infty)$ with

(c) $\dot{x}(t) \in B(t) x(t)+F\left(t, x_{t}\right)$ for a.e. $t \geq s$.

In case the operators $B(t)$ are single-valued, and given the situation that for some $\varphi \in D$ the assertions of steps (1) and (2) of the Introduction hold (with $D=\operatorname{cl} D(A(t))$ independent of $t \geq 0$ ), the following technique is used to prove that the function $x(\varphi ; s)$ defined by $(0.2)$ is a solution to (FDE), provided $X^{*}$ is uniformly convex (cf. $\left.[12,22]\right)$ :

For $\lambda>0$, consider the evolution system $\left\{U_{\lambda}(t, s) \mid 0 \leq s \leq t\right\}$ generated by $\left(A_{\lambda}(t)\right)_{t \geq 0}$ [8]. Then (see [8]),

(1.1) $\lim _{\lambda \rightarrow 0^{+}} U_{\lambda}(t, s) \varphi=U(t, s) \varphi$ uniformly on bounded $t$-intervals, 
and $u_{\lambda}(t)=U_{\lambda}(t, s) \varphi$ is the unique continuously differentiable solution of

$$
\left\{\begin{array}{l}
\dot{u}_{\lambda}(t)+A_{\lambda}(t) u_{\lambda}(t)=0, \quad t \geq s \\
u_{\lambda}(s)=\varphi
\end{array}\right.
$$

(1.2) and the definition of $D(A(t))$ in (0.1) lead to

$$
\begin{aligned}
\left(U_{\lambda}(t, s) \varphi\right)(0)=\varphi(0)+\int_{s}^{t} & {\left[B(\tau)\left(J_{\lambda}(\tau) U_{\lambda}(\tau, s) \varphi(0)\right)\right.} \\
& \left.+F\left(\tau, J_{\lambda}(\tau) U_{\lambda}(\tau, s) \varphi\right)\right] d \tau .
\end{aligned}
$$

Starting from (1.1) and (1.3), the proof is completed by means of the following Lemma of Kato [16] (for details, see [12, Theorem 3]).

Lemma (Kato [16, Lemma 2.5; 1, Chapter II.3, Proposition 3.5]). Assume that $X^{*}$ is uniformly convex and that $C \subset X \times X$ is $m$-accretive, and let $x, y \in X$. If $\left[x_{n}, y_{n}\right] \in C, n \in \mathbb{N}$, and $\left\|x_{n}-x\right\| \rightarrow 0$ and weak-lim $y_{n}=y$, then $[x, y] \in C$.

Whereas steps (1) and (2) of the Introduction work as well for $B(t) \subset X \times X$ multivalued, the above technique for step (3) fails for this case.

In the subsequent section, we derive a parametrized version of Kato's lemma that will remedy this problem.

\section{A PARAMETRIZED VERSION OF KATO'S LEMMA}

Throughout this (and the subsequent section), $X$ will denote a (real) reflexive Banach space whose norm is Fréchet-differentiable at any $x \in X \backslash\{0\}$. The latter condition is equivalent to the duality mapping $J$ of $X$ being single-valued and norm-norm-continuous and is implied by $X^{*}$ being locally uniformly convex (cf. [9, Chapter 2, §2]). Recall that $J: X \rightarrow 2^{X^{*}}$ is defined by $J(x)=\left\{x^{*} \in\right.$ $\left.X^{*} \mid\left\langle x, x^{*}\right\rangle=\|x\|^{2}=\left\|x^{*}\right\|^{2}\right\}, \quad x \in X$.

Lemma 2.1. Assume that, for some $T>0,\{B(\sigma) \mid \sigma \in[0, T]\}$ is a family of (generally, nonlinear and multivalued) operators $B(\sigma) \subset X \times X$ such that, for each $\sigma \in[0, T],(B(\sigma)+\alpha(\sigma) I)$ is maximal accretive for some $\alpha(\sigma) \in \mathbb{R}$ ( $I$ denoting the identity map in $X$ ).

For given $0 \leq s<T$, let $\left(y_{n}\right)_{n} \subset L^{\infty}([s, T] ; X)$ be a bounded sequence, and $x, x_{n}:[s, T] \rightarrow X, n \in \mathbb{N}$, functions from $[s, T]$ into $X$, such that

(i) $\left\|x_{n}(\sigma)-x(\sigma)\right\| \rightarrow 0$ a.e. $\sigma \in[s, T]$, and

(ii) $\left[x_{n}(\sigma), y_{n}(\sigma)\right] \in B(\sigma)$ a.e. $\sigma \in[s, T]$ for all $n \in \mathbb{N}$.

Then there exist $y \in L^{\infty}([s, T] ; X)$ and a subsequence $\left(y_{n_{k}}\right)_{k}$ of $\left(y_{n}\right)_{n}$ such that

(a) $\left(y_{n_{k}}\right)_{k}$ converges to $y$ weakly in $L^{1}([s, T] ; X)$, and

(b) $[x(\sigma), y(\sigma)] \in B(\sigma)$ a.e. $\sigma \in[s, T]$.

Proof. (a) As $X$ is reflexive and $\left(y_{n}\right)_{n}$ is supposed to be $L^{\infty}([s, T] ; X)$ bounded, the vector version of Dunford's classical $L^{1}$-weak compactness criterion [10, Chapter IV.2, Theorem 1] reveals that the set $\left\{y_{n} \mid n \in \mathbb{N}\right\}$ is weakly relatively compact in $L^{1}([s, T] ; X)$. Thus, there exist $y \in L^{1}([s, T] ; X)$ and a subsequence $\left(y_{n_{k}}\right)_{k}$ of $\left(y_{n}\right)_{n}$ that is $L^{1}([s, T] ; X)$-weakly convergent 
to $y$. Clearly, from our assumption of $L^{\infty}([s, T] ; X)$-boundedness of the sequence $\left(y_{n}\right)_{n}$, we have $y \in L^{\infty}([s, T] ; X)$ as well.

(b) Given proposition (a) and replacing the sequence $\left(x_{n}\right)_{n}$ by the subsequence $\left(x_{n_{k}}\right)_{k}$ corresponding to $\left(y_{n_{k}}\right)_{k}$, we can, for the rest of the proof, assume that $\left(y_{n}\right)_{n}$ itself is $L^{1}([s, T] ; X)$-weakly convergent to $y$. Therefore, we have $y \in \bigcap_{n=1}^{\infty} \mathrm{cl} \operatorname{co}\left\{y_{l} \mid l \geq n\right\}$ so that there is a sequence $\left(g^{\prime}{ }_{n}\right)_{n}$ with $g_{n}^{\prime} \in \operatorname{co}\left\{y_{l} \mid l \geq n\right\}$ for $n \in \mathbb{N}$, converging to $y$ in $L^{1}([s, T] ; X)$-norm, and hence a subsequence $\left(g_{n}\right)_{n}$ of $\left(g^{\prime}{ }_{n}\right)_{n}$ converging to $y$ in norm pointwise a.e. on $[s, T]$. Note that $g_{n} \in \operatorname{co}\left\{y_{l} \mid l \geq n\right\}$ for all $n \in \mathbb{N}$ as well so that

$$
g_{n}=\sum_{i=1}^{r_{n}} \alpha_{i}^{n} y_{l(i, n)}
$$

for appropriate $r_{n} \in \mathbb{N}, \alpha_{i}^{n} \geq 0$, and $l(i, n) \geq n$ for all $i \in\left\{1, \ldots, r_{n}\right\}$, and $\sum_{i=1}^{r_{n}} \alpha_{i}^{n}=1$.

Let $E \subset[s, T]$ be a Lebesgue nullset such that $\left\|g_{k}(\sigma)-y(\sigma)\right\| \rightarrow 0$, $\left\|x_{k}(\sigma)-x(\sigma)\right\| \rightarrow 0$ as $k \rightarrow \infty$, and $\left[x_{n}(\sigma), y_{n}(\sigma)\right] \in B(\sigma)$ for all $\sigma \in$ $[s, T] \backslash E$ and all $n \in \mathbb{N}$.

Then, given $\sigma \in[s, T] \backslash E$, we have, for all $[u, v] \in B(\sigma)$,

$$
\begin{aligned}
& \langle y(\sigma)-v, J(x(\sigma)-u)\rangle=\lim _{n \rightarrow \infty}\left\langle g_{n}(\sigma)-v, J(x(\sigma)-u)\right\rangle \\
& =\lim _{n \rightarrow \infty} \sum_{i=1}^{r_{n}} \alpha_{i}^{n}\left\langle y_{l(i, n)}(\sigma)-v, J(x(\sigma)-u)\right\rangle \\
& =\lim _{n \rightarrow \infty}\left\{\sum_{i=1}^{r_{n}} \alpha_{i}^{n}\left\langle y_{l(i, n)}(\sigma)-v, J(x(\sigma)-u)-J\left(x_{l(i, n)}(\sigma)-u\right)\right\rangle\right. \\
& \quad+\sum_{i=1}^{r_{n}} \alpha_{i}^{n}\left\langle y_{l(i, n)}(\sigma)-v, J\left(x_{l(i, n)}(\sigma)-u\right\rangle\right\} \\
& \geq \lim _{n \rightarrow \infty}\left\{-\alpha(\sigma) \sum_{i=1}^{r_{n}} \alpha_{i}^{n}\left\|x_{l(i, n)}(\sigma)-u\right\|^{2}\right\} \\
& =-\alpha(\sigma)\|x(\sigma)-u\|^{2} .
\end{aligned}
$$

Maximal accretivity of $(B(\sigma)+\alpha(\sigma) I)$ implies that $[x(\sigma), y(\sigma)] \in B(\sigma)$ for all $\sigma \in[s, T) \backslash E$. This completes the proof.

\section{Applications to Delay equations}

We first carry out step (3) (as specified in the Introduction) in a general context and then consider particular cases.

As in the foregoing section, we shall consider here (real) reflexive Banach spaces $X$ whose norm is Fréchet-differentiable at any $x \in X \backslash\{0\}$.

\section{A. The general case.}

Assumptions. (a) $E$ is a Banach space of functions from $I$ into $X, I=$ $(-\infty, 0]$, or $I=[-r, 0]$ for some $r>0$, such that the point-evaluation at $\theta=0$ is (well defined and) continuous from $E$ into $X$. 
(b) $(B(t))_{t \geq 0}$ is a family of, generally, multivalued operators $B(t) \subset X \times X$, and $(F(t, \cdot))_{t \geq 0}$ is a family of functions from $E$ into $X$, satisfying:

(B1) For each $t \in[0, \infty)$ there is $\alpha(t) \in \mathbb{R}$ such that $(-B(t)+\alpha(t) I)$ is accretive, $R(I-\lambda B(t))=X$ for $0<\lambda<1 / \max \{0, \alpha(t)\}$, and $\operatorname{cl} D(B(t))$ is independent of $t$; and

(B2) $F(t, \cdot)$ is Lipschitz-continuous with constant $\beta(t) \in \mathbb{R}^{+}, t \geq 0$, such that

(c) for each $T>0, \sup _{t \in[0, T]}\{0, \alpha(t)+\beta(t)\}=\omega(T)<\infty$, and

(d) the family $(A(t))_{t \geq 0}$ of operators defined by $(0.1)$ fulfills the assumptions of [8, Theorem 2.1] for generating an evolution system $\{U(t, s) \mid 0 \leq s \leq t\}$ in $E$, with $\omega$ replaced by $\omega(T)$, and $R(I+\lambda A(t)) \supseteq \operatorname{cl} D=\operatorname{cl} D(A(t))$ for all $0 \leq t \leq T$ and $\lambda>0$ with $\lambda \omega<1$.

(e) $\varphi \in \widehat{D}=\widehat{D}(A(t))$ and $0 \leq s \leq t \leq T$ are such that (0.2), (1.1), and (1.2) are fulfilled, and

(f) for some sequence $0<\lambda_{n} \rightarrow 0$ with $\lambda_{n} \omega(T)<1$ for all $n \in \mathbb{N}$, $\left(\left(A_{\lambda_{n}}(\cdot) U_{\lambda_{n}}(\cdot, s) \varphi\right)(0)\right)_{n}$ and $\left(F\left(\cdot, J_{\lambda_{n}}(\cdot) U_{\lambda_{n}}(\cdot, s) \varphi\right)\right)_{n}$ are uniformly bounded sequences of continuous functions on $[s, T]$. $\infty\}$.)

(Recall [8, Definition 1.1] that $\widehat{D}(A(t))=\left\{\varphi \in \operatorname{cl} D \mid \lim _{\lambda \rightarrow 0^{+}}\left\|A_{\lambda}(t) \varphi\right\|<\right.$

Theorem 3.1. Under the above assumptions 3.A, the function $x(\varphi ; s)$ defined by $(0.2)$ is the unique solution to $(F D E)$.

Proof. Starting from assumptions 3.A, we define functions $x, x_{n}, y_{n}:[s, T]$ $\rightarrow X, \quad n \in \mathbb{N}$, by

$$
x(\tau)=(U(\tau, s) \varphi)(0), \quad x_{n}(\tau)=\left(J_{\lambda_{n}}(\tau) U_{\lambda_{n}}(\tau, s) \varphi\right)(0)
$$

and

$$
y_{n}(\tau)=\left(A_{\lambda_{n}}(\tau) U_{\lambda_{n}}(\tau, s) \varphi\right)(0)+F\left(\tau, J_{\lambda_{n}}(\tau) U_{\lambda_{n}}(\tau, s) \varphi\right),
$$

respectively, $\tau \in[s, T]$. Then we have that $\left(y_{n}\right)_{n}$ is a bounded sequence in $C([s, T] ; X)$ (from (f)), $x \in C([s, T] ; X)$, and $\left\|x_{n}(\sigma)-x(\sigma)\right\| \rightarrow 0$ for all $\sigma \in[s, T]:$ consider

$$
\begin{aligned}
\left\|J_{\lambda_{n}}(\tau) U_{\lambda_{n}}(\tau, s) \varphi-U(\tau, s) \varphi\right\| \leq & \left(1-\lambda_{n} \omega(T)\right)^{-1}\left\|U_{\lambda_{n}}(\tau, s) \varphi-U(\tau, s) \varphi\right\| \\
& +\left\|J_{\lambda_{n}}(\tau) U(\tau, s) \varphi-U(\tau, s) \varphi\right\|
\end{aligned}
$$

and use (1.1), 3.A(d),(e), and [8, Lemma 2.2].

Moreover, by the very definition of $D(A(t))$ in $(0.1),\left[x_{n}(\sigma), y_{n}(\sigma)\right] \in$ $-B(\sigma)$ for all $\sigma \in[s, T]$.

Thus, Lemma 2.1 applies to assert the existence of a subsequence $\left(y_{n_{k}}\right)_{k}$ of $\left(y_{n}\right)_{n}$ that converges weakly in $L^{1}([s, T] ; X)$ to some $y \in L^{\infty}([s, T] ; X)$ such that

$$
[x(\sigma), y(\sigma)] \in-B(\sigma) \text { for a.e. } \sigma \in[s, T] .
$$

According to (1.2) and 3.A(a), we have for all $x^{*} \in X^{*}$

$$
\begin{aligned}
\left\langle U_{\lambda_{n_{k}}}(t, s) \varphi(0), x^{*}\right\rangle= & \left\langle\varphi(0), x^{*}\right\rangle-\int_{s}^{t}\left\langle y_{n_{k}}(\tau), x^{*}\right\rangle d \tau \\
& +\int_{s}^{t}\left\langle F\left(\tau, J_{\lambda_{n_{k}}}(\tau) U_{\lambda_{n_{k}}}(\tau, s) \varphi\right), x^{*}\right\rangle d \tau
\end{aligned}
$$


for all $t \in[s, T]$. Now we use (1.1) for the left side of (3.3), weak convergence in $L^{1}([s, T] ; X)$ of $\left(y_{n_{k}}\right)_{k}$ to $y$ for the second term on the right of (3.3), and (3.1) and uniform boundedness of $\left(F\left(\cdot, J_{\lambda_{n}}(\cdot) U_{\lambda_{n}}(\cdot, s) \varphi\right)\right)_{n}$ on $[s, T]$ and Lebesgue's Dominated Convergence Theorem for the last term on the right of (3.3) to conclude that

$$
U(t, s) \varphi(0)=\varphi(0)-\int_{s}^{t} y(\tau) d \tau+\int_{s}^{t} F(\tau, \quad U(\tau, s) \varphi) d \tau
$$

Hence, by (0.2), we have

$$
x(\varphi ; s)(t)=\varphi(0)-\int_{s}^{t} y(\tau) d \tau+\int_{s}^{t} F\left(\tau, x_{\tau}(\varphi ; s)\right) d \tau
$$

for all $t \in[s, T]$. This shows that the function $x(\varphi ; s)$ as defined in $(0.2)$ is locally absolutely continuous and differentiable a.e. on $[s, \infty)$ and fulfills (by (3.2))

$$
\dot{x}(\varphi ; s)(t)-F\left(t, \quad x_{t}(\varphi ; s)\right) \in B(t) x(\varphi ; s)(t)
$$

for a.e. $t \in[s, \infty)$, i.e., $x(\varphi ; s)$ is a solution to (FDE). This completes the proof of Theorem 3.1 .

\section{B. Examples.}

3.B.1. Finite delay. Here, we take up the examples considered in $[12,14]$ and extend [12, Theorem 3] to the multivalued case.

Assumptions. For $r>0$, let $E=C([-r, 0], X)$ with sup-norm. Let $(B(t))_{t \geq 0}$ and $(F(t, \cdot))_{t \geq 0}$ be families of operators $B(t) \subset X \times X$ and $F(t, \cdot): E \rightarrow$ $X$, with $\mathrm{cl} D(B(t))$ independent of $t \geq 0$, and satisfying $3 . \mathrm{A}(\mathrm{b}), 3 . \mathrm{A}(\mathrm{c})$, and

(B3) For all $T>0$, there exist a continuous function $h:[0, T] \rightarrow X$ of bounded variation, and monotonically increasing functions $L_{1}, L_{2}: \mathbb{R}^{+} \rightarrow \mathbb{R}^{+}$ such that

(i) $\left\|(I-\lambda B(t))^{-1} x-(I-\lambda B(\tau))^{-1} x\right\| \leq \lambda\|h(t)-h(\tau)\| L_{1}(\|x\|)$ for all $x \in X$ and $0 \leq \tau, t \leq T$ and all $\lambda>0$ sufficiently small, and

(ii) $\|F(t, \varphi)-F(\tau, \varphi)\| \leq\|h(t)-h(\tau)\| L_{2}(\|\varphi\|)$ for all $0 \leq \tau, t \leq T$ and all $\varphi \in E$.

Theorem 3.2. Under the above assumptions 3.B.1, the operators $(A(t))_{t \geq 0}$ defined by (0.1) generate an evolution system $\{U(t, s) \mid 0 \leq s \leq t\}$ in $E$ such that, given any $\varphi \in \widehat{D}=\widehat{D}(A(t))$, the function $x(\varphi ; s)$ defined by $(0.2)$ is the unique solution to $(F D E)$.

Moreover, if $\alpha(t)+\beta(t) \leq 0$ for all $t \geq 0$, then the solutions to (FDE) are stable in the sense that, for any two initial histories $\varphi, \psi \in \widehat{D}$, the corresponding solutions $x(\varphi ; s)$ and $x(\psi ; s)$ fulfill

$$
\|x(\varphi ; s)(t)-x(\psi ; s)(t)\| \leq\|\varphi-\psi\| \text { for all } t \geq s .
$$

Proof. For the first part of the result, we have to show that assumptions 3.A(d)-(f) are fulfilled. The last part then follows from [8, Theorem 2.1].

3.A(d) follows along the lines of the argument of $[12, \S 3 ; 14$, Theorem 12 , proof of (i) implies (ii)].

3.A(e), in turn, follows from 3.A(d) and [8, §4]. 
We turn to 3.A(f). Let $\lambda>0$ such that $\lambda \omega(T)<1$, and let $\xi, \xi_{\circ} \in[s, T]$ and $\omega:=\omega(T)$. Then we have

$$
\begin{aligned}
\| A_{\lambda}(\xi) & U_{\lambda}(\xi, s) \varphi-A_{\lambda}\left(\xi_{\circ}\right) U_{\lambda}\left(\xi_{\circ}, s\right) \varphi \| \\
\leq & \left\|A_{\lambda}(\xi) U_{\lambda}(\xi, s) \varphi-A_{\lambda}(\xi) U_{\lambda}\left(\xi_{\circ}, s\right) \varphi\right\| \\
& +\left\|A_{\lambda}(\xi) U_{\lambda}\left(\xi_{\circ}, s\right) \varphi-A_{\lambda}\left(\xi_{\circ}\right) U_{\lambda}\left(\xi_{\circ}, s\right) \varphi\right\| \\
\leq & \lambda^{-1}\left(1+(1-\lambda \omega)^{-1}\right)\left\|U_{\lambda}(\xi, s) \varphi-U_{\lambda}\left(\xi_{\circ}, s\right) \varphi\right\| \\
& +\left\|h(\xi)-h\left(\xi_{\circ}\right)\right\| L\left(\left\|U_{\lambda}\left(\xi_{\circ}, s\right) \varphi\right\|\right)
\end{aligned}
$$

for some monotonically increasing function $L: \mathbb{R}^{+} \rightarrow \mathbb{R}^{+}$. (Here we have used 3.A(d) and [8, Lemma 1.2; 14, Theorem 12].) We have thus shown that $\left(A_{\lambda}(\cdot) U_{\lambda}(\cdot, s) \varphi\right)(0)$ is continuous on $[s, T]$.

Next, using (B3)(ii), it is easy to check that, for any sequence $0<\lambda_{n} \rightarrow$ 0 with $\lambda_{n} \omega(T)<1$ for all $n \in \mathbb{N},\left(F\left(\cdot, J_{\lambda_{n}}(\cdot) U_{\lambda_{n}}(\cdot, s) \varphi\right)\right)_{n}$ is a uniformly bounded sequence of continuous functions on $[s, T]$ for all $n$ large enough (depending only on $s$ and $T$ ).

At this point, recall that $u_{\lambda}(t)=U_{\lambda}(t, s) \varphi$ is the unique continuously differentiable solution of (1.2). Hence, we have

$$
u_{\lambda}(t)=\exp \left(-\lambda^{-1}(t-s)\right) \varphi+\lambda^{-1} \exp \left(-\lambda^{-1} t\right) \int_{s}^{t} \exp (\xi / \lambda) J_{\lambda}(\xi) u_{\lambda}(\xi) d \xi
$$

Following the arguments of [7, Proof of Theorem 1.5, pp. 13-14], we arrive at

$$
\left\|\dot{u}_{\lambda}(\xi)\right\| \leq\left\|\dot{u}_{\lambda}(s)\right\|+C V_{s}^{T}[h],
$$

for all $\lambda=\lambda_{n}$ with $n$ so large that $\left(U_{\lambda_{n}}(\cdot, s) \varphi\right)_{n}$ is uniformly bounded on $[s, T]$ by $C_{1}>0 .\left(V_{s}^{T}[h]\right.$ is the total variation of $h$ on $[s, T]$, and $C=L\left(C_{1}\right)$.) Using [8, Lemma 1.3], we conclude from (3.5) that

$$
\left\|A_{\lambda_{n}}(\xi) U_{\lambda_{n}}(\xi, s) \varphi\right\| \leq\left(1-\lambda_{n} \omega\right)^{-1}|A(s) \varphi|+C V_{s}^{T}[h]
$$

for all $\xi \in[s, T]$ and all $n$ large enough. But $|A(s) \varphi|=\lim _{\lambda \rightarrow 0^{+}}\left\|A_{\lambda}(s) \varphi\right\|$ $<\infty$ for $\varphi \in \widehat{D}=\widehat{D}(A(t)), t \geq 0$. We have thus shown that $3 . \mathrm{A}(\mathrm{f})$ is fulfilled, and the proof of Theorem 3.2 is complete.

3.B.2. Infinite delay. Theorem 3.2 holds as well in the case of infinite delay, more precisely, in case the initial history space is taken to be $E=\mathrm{BUC}\left(\mathbb{R}^{-}, X\right)$, the space of bounded uniformly continuous functions from $(-\infty, 0]$ into $X$, endowed with the sup-norm (consult $[14, \S 6 ; 18$, Theorem 3.1]).

3.B.3. The autonomous case. In [19] we consider the autonomous case of (FDE), i.e., with $B=B(t)$ and $F=F(t, \cdot)$ independent of $t \geq 0$, for

(a) more general classes of initial history spaces $E$ (fulfilling assumption 3.A(a)), and

(b) most importantly, for functions $F: E \rightarrow X$ which are only locally Lipschitz-continuous. (This is crucial for concrete applications in population dynamics for instance.)

The general technique for step (3) as presented at the beginning of this section works as well in these situations, leading to results corresponding to Theorem 3.2; see [19]. 
3.B.4. We close this paper by a concrete example for the case of multivalued operators $(B(t))_{t \geq 0}$.

As follows from the discussion in [11, Chapter I.2.3] and corresponding results in [6, Corollary 28], considering a continuous medium occupying an open region $\Omega$ of $\mathbb{R}^{n}$, temperature control regulated by the temperature in the interior is governed by the operator

$$
B=-\Delta+\tilde{\beta} \quad \text { with } D(B)=H^{2}(\Omega) \cap H_{\circ}^{1}(\Omega) \cap D(\tilde{\beta}),
$$

where $\beta \subset \mathbb{R} \times \mathbb{R}$ is maximal monotone with $0 \in D(\beta)$ and $\tilde{\beta}$ is the realization of $\beta$ in $H=L^{2}(\Omega)$ :

$$
\begin{gathered}
D(\tilde{\beta})=\{u \in H \mid \text { there exists } v \in H \text { with } v(x) \in \beta(u(x)) \text { a.e. } x \in \Omega\}, \\
\tilde{\beta}(u)=\{v \in H \mid v(x) \in \beta(u(x)) \text { a.e. } x \in \Omega\}, \quad u \in D(\tilde{\beta}) .
\end{gathered}
$$

Theorem 3.2 and its infinite-delay counterpart 3.B.2 thus apply to problems of time-dependent temperature control, or pressure control for fluid in a porous medium (cf. [11, Chapter I.2] for these and related problems in physics), with $\beta(t) \subset \mathbb{R} \times \mathbb{R}$ suitably depending on $t \geq 0$, taking into account the history of the process. For the operator $B$ in the general $L^{p}(\Omega)$-context, $1<p<\infty$, the reader is referred to [1, Chapter II.3.2, Proposition 3.7].

\section{ACKNOWLEDGMENT}

The problem considered in this paper grew out of a joint project with $\mathrm{W}$. $\mathrm{H}$. Summers on the asymptotic behavior of solutions to evolution equations (supported in part by DFG and NSF). I am grateful to Bill Summers and Ph. Bénilan for helpful discussions on the subject of this paper.

\section{REFERENCES}

1. V. Barbu, Nonlinear semigroups and differential equations in Banach spaces, Noordhoff, Leyden, 1976.

2. D. W. Brewer, A nonlinear semigroup for a functional differential equation, Trans. Amer. Math. Soc. 236 (1978), 173-191.

3. Equations (S.-O. Londen and O. J. Staffans, eds.), Lecture Notes in Math., vol. 737, Springer, New York, 1979, pp. 35-44.

4. delay, Houston J. Math. 6 (1980), 321-330.

5. L Locally Lipschitz continuous functional differential equations and nonlinear semigroups, Illinois J. Math. 26 (1982), 374-381.

6. H. Brézis, Monotonicity methods in Hilbert spaces and some applications to nonlinear partial differential equations, Contributions to Nonlinear Functional Analysis (E. Zarantonello, ed.), Academic Press, New York, 1971, pp. 101-156.

7. Opérateurs maximaux monotones et semigroupes de contractions dans les espaces de Hilbert, North-Holland Math. Studies, vol. 5, North-Holland, Amsterdam, 1973.

8. M. G. Crandall and A. Pazy, Nonlinear evolution equations in Banach spaces, Israel J. Math. 11 (1972), 57-94.

9. J. Diestel, Geometry of Banach spaces-selected topics, Lecture Notes in Math., vol. 485, Springer, New York, 1975.

10. J. Diestel and J. J. Uhl, Vector measures, Math. Surveys Monographs, vol. 15, Amer. Math. Soc., Providence, RI, 1977. 
11. G. Duvaut and J. L. Lions, Inequalities in mechanics and physics, Grundlehren Math. Wiss., vol. 219, Springer, New York, 1976.

12. J. Dyson and R. Villella Bressan, Functional differential equations and non-linear evolution operators, Proc. Roy. Soc. Edinburgh Sect. A 75 (1975/76), 223-234.

13. __ Some remarks on the asymptotic behavior of a nonautonomous, nonlinear functional differential equation, J. Differential Equations 25 (1977), 275-287.

14. __ Semigroups of translation associated with functional and functional differential equations, Proc. Roy. Soc. Edinburgh Sect. A 82 (1979), 171-188.

15. H. Flaschka and M. J. Leitman, On semigroups of nonlinear operators and the solution of the functional differential equation $\dot{x}(t)=F\left(x_{t}\right)$, J. Math. Anal. Appl. 49 (1975), 649-658.

16. T. Kato, Nonlinear semigroups and evolution equations, J. Math. Soc. Japan 19 (1967), 508520.

17. N. Pavel, Nonlinear evolution operators and semigroups, Lecture Notes in Math., vol. 1260, Springer, New York, 1987.

18. A. T. Plant, Nonlinear semigroups of translations in Banach space generated by functional differential equations, J. Math. Anal. Appl. 60 (1977), 67-74.

19. W. M. Ruess and W. H. Summers, Operator semigroups for functional differential equations with delay, Trans. Amer. Math. Soc. (to appear).

20. C. C. Travis and G. F. Webb, Existence and stability for partial functional differential equations, Trans. Amer. Math. Soc. 200 (1974), 395-418.

21. G. F. Webb, Autonomous nonlinear functional differential equations and nonlinear semigroups, J. Math. Anal. Appl. 46 (1974), 1-12.

22. Amer. Math. Soc. 54 (1976), 225-230.

Fachbereich Mathematik, Universität Essen, D-45117 Essen, Federal Republic of GERMANY

E-mail address: mate00@vm.hrz.uni-essen.de 